\title{
Zweidimensionale Arraytransducer für die medizinische Ultraschalldiagnostik - was bringt die Zukunft?
}

\author{
Moser, U
}

DOI: https://doi.org/10.1515/bmte.1996.41.s1.166

Posted at the Zurich Open Repository and Archive, University of Zurich ZORA URL: https://doi.org/10.5167/uzh-154359

Journal Article

Published Version

Originally published at:

Moser, U (1996). Zweidimensionale Arraytransducer für die medizinische Ultraschalldiagnostik - was bringt die Zukunft? Biomedizinische Technik. Biomedical engineering, 41(s1):166-169.

DOI: https://doi.org/10.1515/bmte.1996.41.s1.166 


\title{
Zweidimensionale Arraytransducer für die medizinische Ultraschalldiagnostik - was bringt die Zukunft?
}

\author{
U. Moser \\ Institut für Biomedizinische Technik und Medizinische Informatik, \\ ETH und Universität Zürich, CH-8092 Zürich
}

\section{Einleitung}

Die stetig erzielten Fortschritte der Ultraschalldiagnostik während der letzten Jahre sind das Resultat sich gegenseitig stimulierender Entwicklungen, die parallel auf verschiedenen Ebenen ablaufen:

- In konzeptioneller Hinsicht wurden die Ultraschall-Geräte vom einfachen Echogerät mit mechanischer Strahlauslenkung zum komplexen Farb-Duplexgerät mit Array-Transducer weiter entwickelt. Immer wieder bringen innovative Ideen Verbesserungen und neue Anwendungsmöglichkeiten, wie z.B. der Tissue- und Powerdoppler, die 3d-Technik oder die parallele Datenakquisition.

- Verbesserte Algorithmen und neue Methoden bei der Signal- und Bildverarbeitung brachten eine vollständigere Auswertung der in den Ultraschallechos enthaltenen Information. Damit wurden sowohl bei der Echographie als auch der Blutflussmesstechnik (Doppler) Fortschritte erzielt.

- Schliesslich hat der technologische Fortschritt ebenfalls wesentlich zur Qualitätsverbesserung beigetragen. Verbesserte Piezomaterialien und verfeinerte Herstellungsverfahren haben die Effizienz der Schallwandler (Transducer) erheblich gesteigert. Nicht zuletzt hat die rasche Entwicklung der Mikorelektronik (Digitaltechnik) neue Möglichkeiten geschaffen. Kostengünstige und sehr leistungsfähige Signalprozessoren erlauben das Implementieren aufwendiger Algorithmen für die EchtzeitSignalverarbeitung. Die stark gesunkenen Kosten der Elektronik-Bauteile erlauben mehr und mehr den kommerziellen Einsatz vieler paralleler Sende- und Empfangskanäle, wie sie zum Betrieb künftiger zweidimensionaler Arraytransducer zu hunderten oder tausenden notwendig sein werden. $\mathrm{Zu}$ erwähnen ist ausserdem die heute zur Verfugung stehende leistungsfähige Software, dank der insbesondere bei der Bildverarbeitung Fortschritte zu verzeichenen sind.

Die Summe dieser Entwicklungen resultiert in einer laufenden Verfeinerung der Ultraschalldiagnostik. Sie fuiht aber auch zu einer immer komplexeren Technik, die eine breite Palette interdisziplinärer Kenntnisse für die Entwicklung, Produktion und Anwendung moderner Ultraschallgeräte voraussetzt. Die unbestrittenen
Vorteile der Ultraschalldiagnostik wie Patientenfreundlichkeit, Echtzeitfähigkeit, vergleichsweise geringe Kosten etc., motivieren weiterhin das Bestreben, die Bildqualität und die Messgenauigkeit dieses nicht-invasiven Verfahrens bis an die von der Physik gesetzten Grenzen zu steigern.

Mit den heute verwendeten Transducer-Typen ist dieses Ziel jedoch nicht zu erreichen. Eindimensionale Linearund Phased-Array-Transducer erlauben z.B. nur eine elektronische Fokussierung in der Scan-Ebene. Besonders nachteilig ist, dass die Ortsinformation über die Schalldruckverteilung senkrecht zur Scan-Ebene verloren geht, da über die Fläche der Arrayelemente eine Mittelung stattfindet. Annuläre Arrays ermöglichen zwar eine rotationssymmetrische dynamische Fokussierung, es besteht aber der gleiche Mittelungseffekt entlang der Ringelemente.

Nachfolgend einige Überlegungen und Beispiele, weshalb in Zukunft 2d-Arrays in verschiedenster Form trotz des beträchtlichen technischen Aufwands mehr und mehr zum Einsatz kommen werden.

\section{Das Problem der Laufzeitverzerrungen}

Weshalb ist es wichtig, die zweidimensionale Schalldruckverteilung in der Transducer-Apertur zu kennen?

Das theoretische Auflösungsvermögen in einer bestimmten Distanz ist aufgrund der Beugung im wesentlichen durch das Verhältnis Transducergrösse/Wellenlänge bestimmt. Ein besseres Auflösungsvermögen kann praktisch nur noch durch Vergrössern der Transducer-Apertur erreicht werden, da die Gewebedämpfung exponentiell mit der Sendefrequenz zunimmt. Das beugungslimitierte Auflösungsvermögen wird im Gewebe jedoch mit den heute verwendeten Transducertypen nicht erreicht, da die unterschiedlichen Schallgeschwindigkeiten der verschie- denen Gewebearten zu Laufzeitfehlern führt, die eine Verschmierung des Fokus zur Folge haben. Dieser Effekt ist umso ausgeprägter, je grösser das Verhältnis der Apertur zur Fokaldistanz und je kürzer die verwendete Wellenlänge ist, d.h. je mehr versucht wird, das Auflösungsvermögen zu steigern. Fig. 1 erläutert diesen Sachverhalt.

Es ist nur denkbar, diese Laufzeitfehler zu korrigieren, wenn die zweidimensionale Schalldruckverteilung auf dem Transducer bekannt ist. Weder bei 
eindimensionalen noch bei annulären Arrays ist dies jedoch der Fall.

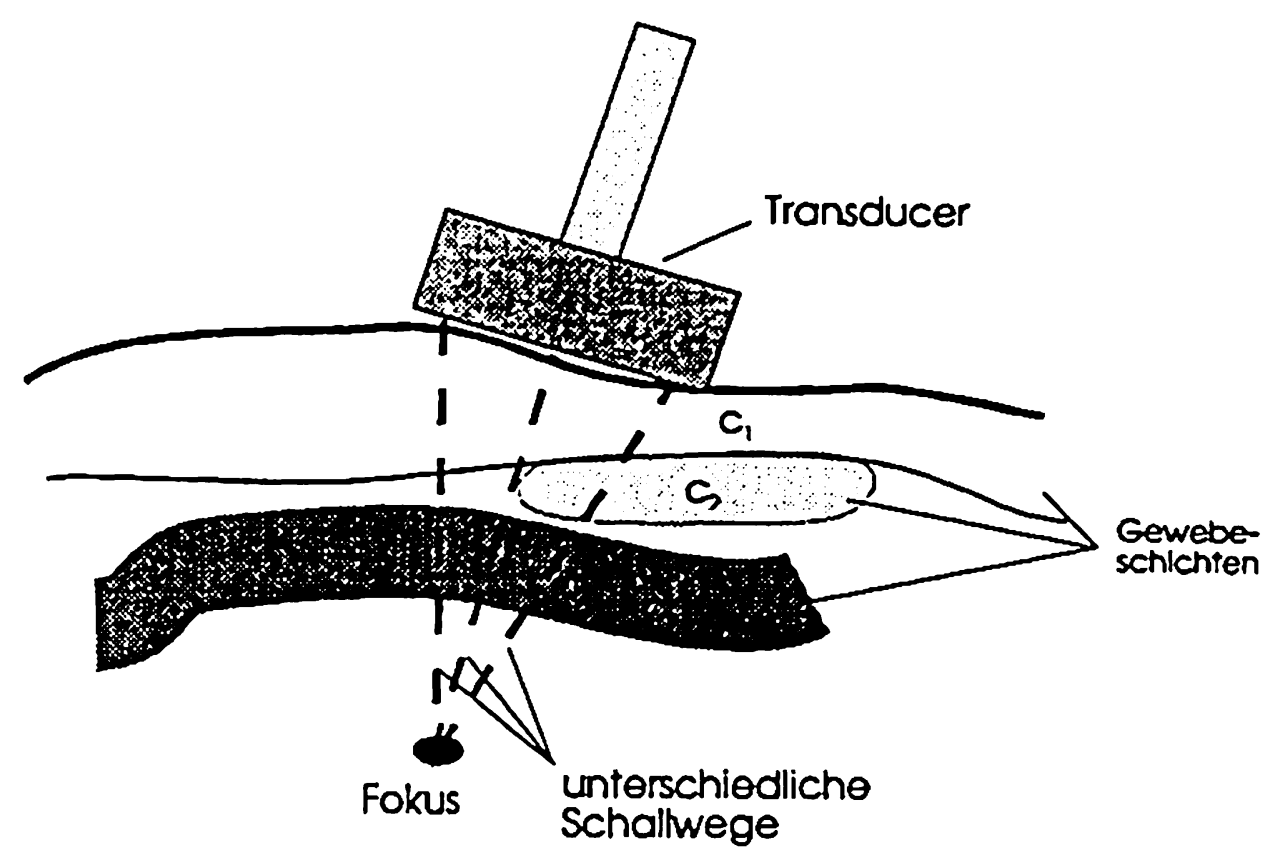

Fig. 1: Schallwege zwischen Transducerfläche und Brennpunkt. Führen diese durch Gebiete mit unterschiedlicher Schallgeschwindigkeit, wird der geometrische Brennpunkt verschmiert. Durch Vergrössern des Transducers oder Verkleinern der Wellenlänge kann die gewünschte Verbesserung des Auflösungsvermögens nicht ereicht werden, da der Verschmierungseffekt mehr und mehr dominiert.

\section{Der 2d-Array als Ausweg?}

Ein 2d-Array ist zusammen mit einer parallelen Empfangselektronik in der Lage, die gesamte Information zu registrieren, die der beim Transducer ankommende rückgestreute Ultraschall enthält. Das heisst, dass Phase und Amplitude des Schalldruckes in der Apertur örtlich und zeitlich bekannt sind. Dies ist die Voraussetzung für folgende Massnahmen:

- zweidimensionale dynamische Fokussierung

- Ultraschnelle Datenakquisition durch paralleles Beobachten mehrerer Bereiche (Voxel), z.B. für genauere 2d-Doppler Messungen

- Korrektur der durch die verschiedenartigen Gewebeschichten venursachten Laufzeitverzerrungen und damit Verbesserung des Fokus

Der erste Punkt kann bei entsprechendem Aufwand an programmierbaren Sende- und Empfangskanälen relativ leicht realisiert werden. Wird die Strahlauslenkung konventionell auf eine Ebene beschränkt, dann kann der Transducer senkrecht zur Scan-Richtung ziemlich grob unterteilt werden. Diese grobe Unterteilung dient nur zur elektronischen Fokussienung. Ein entsprechender Array könnte z.B. aus $128 \times 5$ Elementen bestehen, so dass 640 Sende-Empfangskanäle notwendig wären.

Der 2. Punkt dürfte hauptsächlich bei der Dopplertechnik von Bedeutung sein, da eine lange Beobachtungszeit die Stabilität der Geschwindigkeitsmessung verbessert. Ein Beispiel stellt der weiter unten beschriebene C-mode Doppler dar.

Der letzte Punkt gilt als anspruchvollstes Vorhaben. Da die Verteilung der Gewebeschichten mit ihren unterschiedlichen Schallgeschwindigkeiten a priori unbekannt ist, ist die Laufzeitkorrektur kein leichtes Unterfangen. Nur in Spezialfällen ist dies relativ einfach möglich, etwa bei vorhandenem starken Punktreflektor (z.B. Nieren- oder Gallenstein) [1,2]. Im allgemeinen muss jedoch mit Hilfe des lokalen Kontrastes oder anderer Kriterien optimiert werden.

Je nach Anwendung stellt sich die Frage, wie viele Arrayelement minimal notwendig sind. Das Registrieren der Schalldruckverteilung mit Hilfe der einzelnen Transducerelemente kann als räumliches Abtasten interpretiert werden. Bei der Wahl der Grösse der Arrayelemente ist deshalb das Nyquisttheorem zu berücksichtigen. Um in jedem Fall störende Effekte wie Gitterkeulen zu vermeiden, müssen die Transducerelemente kleiner sein als eine halbe Wellenläge. Es ist jedoch leicht nachzurechnen, dass der Bau eines entsprechenden 2d-Arrays sowohl fertigungstechnisch als auch aufgrund der benötigten Elektronik bis auf weiteres eine beachtliche technische Herausforderung darstellt. Für einen quadratischen 2d-Array mit $20 \mathrm{~mm}$ Kantenlänge und $0.4 \mathrm{~mm}$ Wellenlänge (3.5MHz) benötigte man z.B. $10^{\prime} 000$ Transducerelemente!

\section{2d-Arrays mit grossen Elementen - ein kostengünstiger Kompromiss}

Im folgenden wird gezeigt, dass auch 'grobe' 2d-Arrays (Abmessungen der Elemente beträgt mehrere Wellenlängen) für verschiedene Anwendungen interressant sind. Dazu müssen zuerst die Eigenschaften solcher Arrays, insbesondere das Entstehen von Gitterkeulen, betrachtet werden. Die Schwierigkeit dabei ist, dass Nahfeldeffekte eine wichtige Rolle spielen oder sogar die Feldcharakteristik dominieren, so dass die bekannten Fernfeldapproximationen nur in bestimmten Bereichen Gültigkeit haben. Wir betrachten dazu Bild 2. Das Schallfeld eines 'groben' 2d-Array s lässt sich in 3

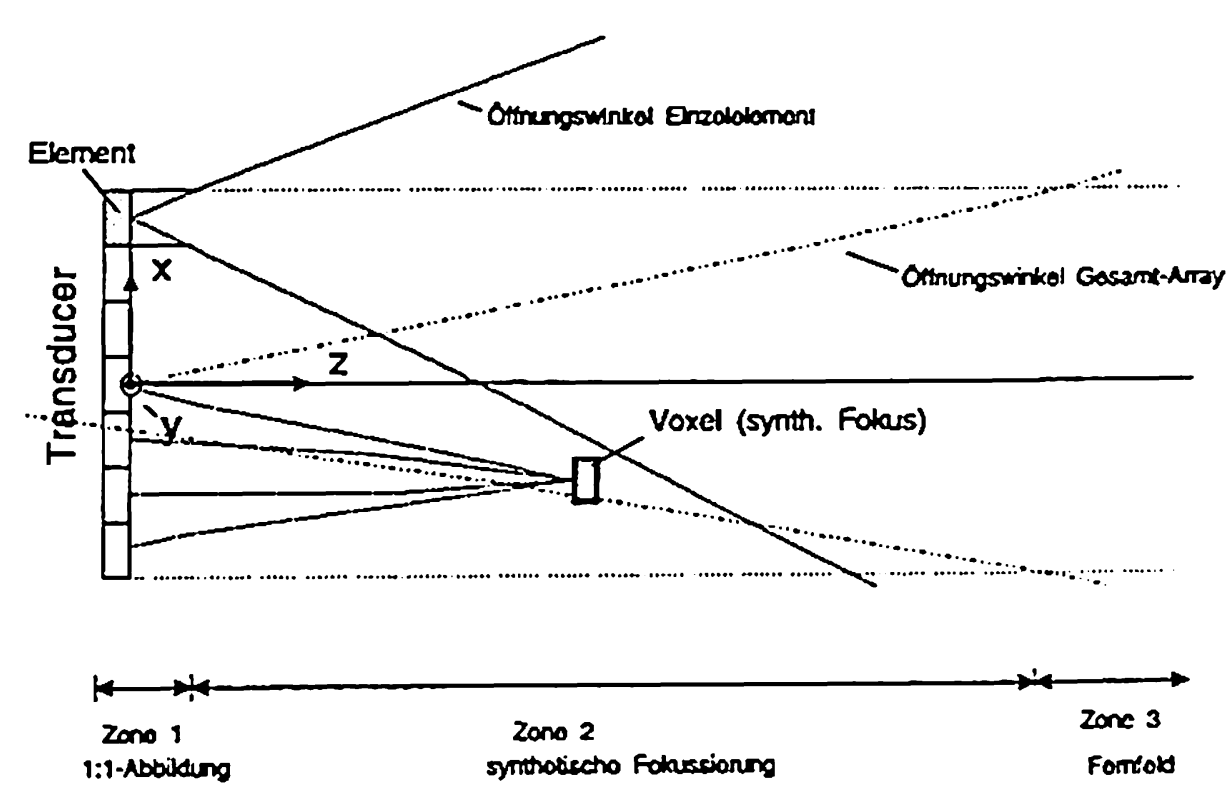

Fig. 2: Charakterisierung des Schallfeldes eines Arrays mit grossen Elementen. Zone 1: Jedes Transducerelement beschallt das unmittelbar vor ihm liegende Gebiet. Zone 2: Mit zunehmender Distanz können mehr und mehr Elemente zur synthetischen Fokussierung verwendet werden. Zone 3: Fernfeld des Arrays. 
Zonen aufteilen. Die 1. Zone stellt das Nahfeld eines einzelnen Transducerelementes dar. Das laterale Auflösungsvermögen entspricht dem Elementabstand, es ist nur eine 1:1 Abbildung möglich. Dieser Distanzbereich hat allenfalls bei Doppleranwendungen eine Bedeutung. Die 2. Zone beginnt ab dem Fernfeld eines Einzelelementes und endet beim übergang Nahfeld-Fernfeld des Gesamt-Arrays. Normalerweise befindet sich der Einsatzbereich in dieser Zone. Allgemein kann hier das Schallfeld nur mittels aufivendiger Computersimulation berechnet werden. Wird das Feld jedoch fokussiert, was bei bildgebenden Verfahren in der Regel der Fall ist, dann gelten im Fokalbereich die selben Näherungsformeln wie im Fernfeld. Allerdings nur, wenn diejenigen Transducerelemente, die am Fokus 'vorbeizielen', ausgeblendet werden. Dies kann je nach Abstand des Fokus vom Transducer und der z-Achse mit einer breiteren oder schmaleren Fensterfunktion zur Gewichtung der Arrayelemente bewerkstelligt werden (dynamische Apertur). Als Aperturfunktion hat sich z.B. eine Gauss-Funktion bewährt.

Die 3. Zone schliesslich entspricht dem Fernfeld des Arrays. Bei den hier anvisierten Arrays mit grosser Apertur liegt dieser Bereich ausserhalb der üblichen Einsatzdistanz.

Arrays mit grober Unterteilung und entsprechend wenig Elementen eignen sich kaum, um das Schallfeld elektronisch auszulenken, es sei denn, in einem ganz limitierten Bereich. Die dabei entstehenden Gitterkeulen verhindern normalerweise deren Anwendung in der Echographie. Fig. 3 zeigt das berechnete Schallfeld eines $8 \times 8$ Arrays in einer Ebene durch den Fokus in $80 \mathrm{~mm}$ Distanz. Die Schallkeule wurde von der Transducerachse elektronisch ausgelenkt. Dadurch entstehen Gitterkeulen, die fast die Hälfte der Hauptkeule erreichen. Mittels Gewichtung der Amplituden der Arrayelemente lassen sich die Gitterkeulen auf ein für spezielle Anwendungen tolerierbares Mass dämpfen. Allerdings wird dadurch die Hauptkeule verbreitert.

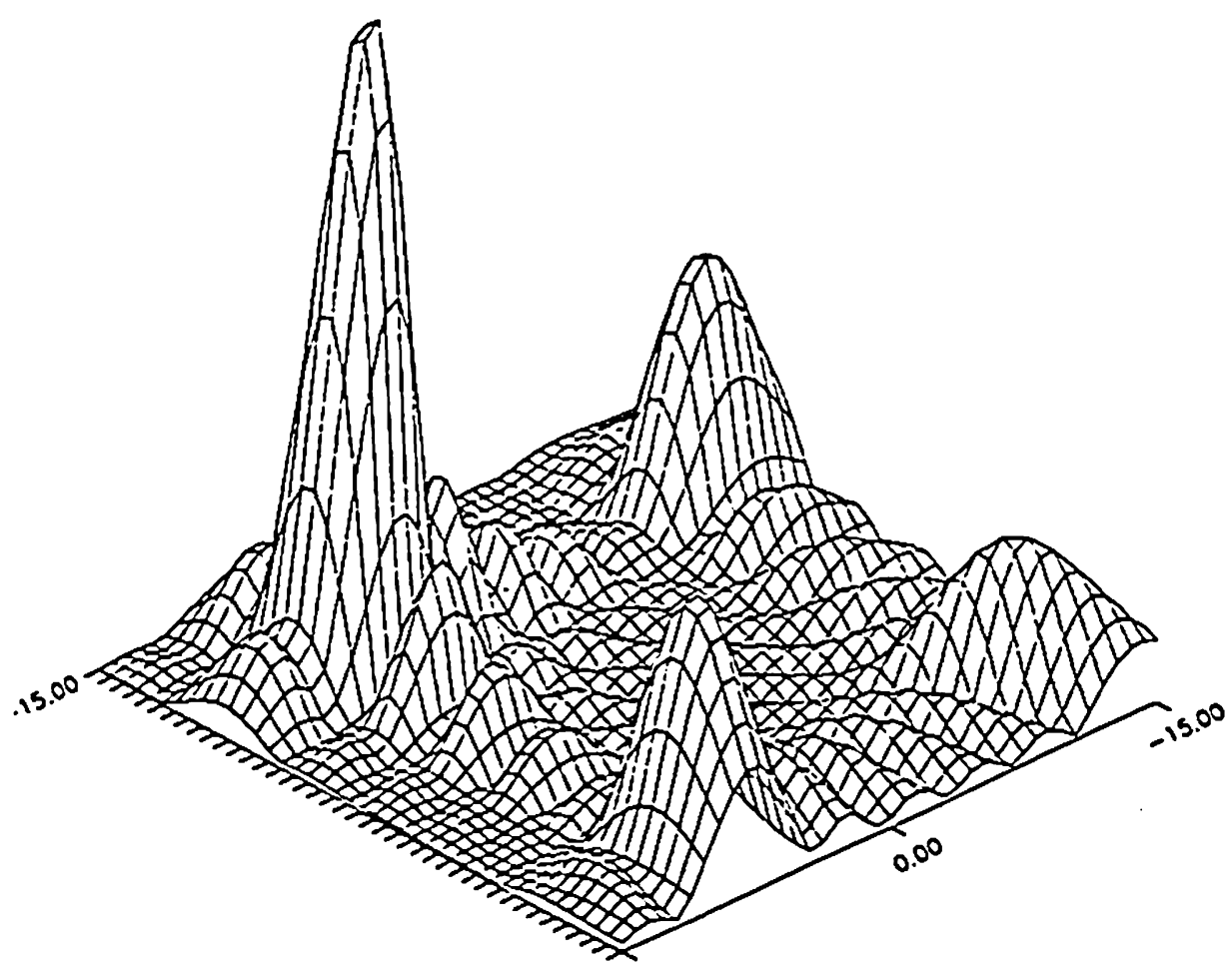

Fig. 3: Schallfeld eines 'groben' 2d-Arrays (8x8 mit $12 \mathrm{~mm}$ Kantenlänge, $4 \mathrm{MHz}$ ) mit 5.5 Grad elektronischer Strahlauslenkung in Azimuth und Elevation und Fokussierung in $80 \mathrm{~mm}$ Distanz. Die Gitterkeulen sind für die meisten Anwendungen nicht tolerierbar (Simulation P.M.Schumacher).
5.Grober 2d-Array in der Dopplertechnik: C-Mode als Beispiel

Der C-mode Doppler ist ein Beispiel dafuir, dass auch 2d-Arrays mit wenigen Elementen nützlich sein können. Das Verfahren wurde entwickelt, um in grossen Blutgefässen das zweidimensionale Strömungsfeld und den Volumenfluss in Echtzeit zu messen. Fig. 4 zeigt das Prinzip. Die Messschicht steht senkrecht zur Strahlrichtung und deckt den Querschnitt des zu untersuchenden Gefässes vollständig ab.

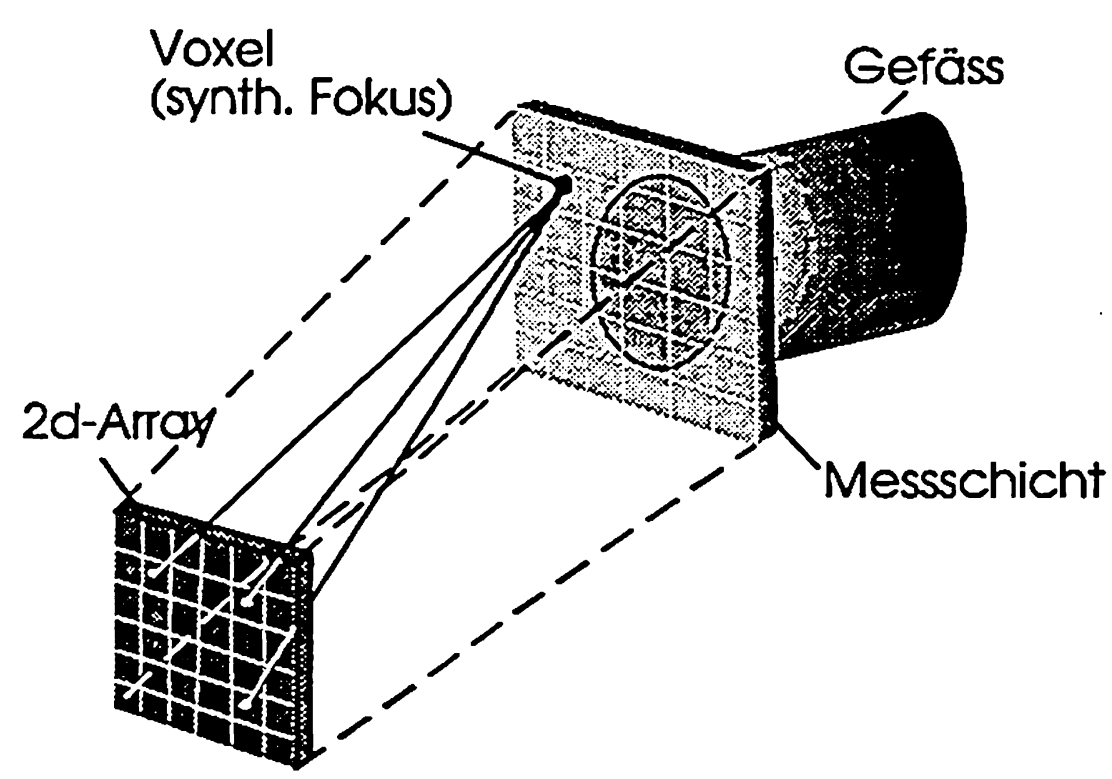

Fig. 4: Anordnung von 2d-Array und Messschicht beim C-Mode Doppler. Das Verfahren erlaubt, innerhalb von 10 - 30ms die zweidimensionale Stromungsverteilung in grossen Blutgefässen und den Volumenfluss zu messen.

Daher ist das interessierende Gebiet relativ klein und es genügen wenige Messpunkte. Die Dynamikanforderungen an den Array sind ebenfalls bescheiden, (d. h. das Verhältnis von Haupt- zu Neben- bzw. Gitterkeulen muss nicht besonders gross sein), da das vom Blut reflektierte Dopplersignal eine vergleichsweise konstante Stärke besitzt. Die von den übrigen Gewebestrukturen erzeugten Echos weisen zwar grosse Dynamikunterschiede auf, sie besitzen jedoch keine Dopplerverschiebung und können mittels Stationärechofiltern unterdrückt werden. Zudem stören die Gitterkeulen auch deshalb wenig, weil beim Senden die Schallenergie hauptsächlich im interessierenden gitterkeulen-freien Bereich deponiert wird.

Dank dem 2d-Array ist es möglich, die in der Messschicht liegenden Voxel alle gleichzeitig zu beobachten. Zu diesem Zweck wird beim Senden mit Hilfe eines breiten Schallpulses von etwa $1 \mu$ s Dauer der interessierende Bereich gesamthaft ausgeschallt. In der Empfangsphase werden die Signale der Transducerelemente parallel digitalisiert und zwischengespeichert. Aus diesem Datensatz kann anschliessend mittels synthetischer Fokussierung eine grosse Anzahl Voxel in der Messchicht separiert werden. Dieser Vorgang wird periodisch wiederholt, wobei die Pulsfolgefrequenz wie in der Puls-Dopplertechnik üblich, $5-10 \mathrm{kHz}$ beträgt. Für die - synthetische Fokussierung stehen somit lediglich $100-200 \mu$ s zur Verfügung. Werden nun die so separierten Daten mehrerer Pulsperioden einer Frequenzanalyse unterzogen, kann daraus die mittlere Geschwindigkeit in 
den einzelnen Voxeln bestimmt werden. Das gleichzeitige Beobachten der Voxel eliminiert das Problem der knappen Beobachtungszeit, wie es z.B. beim Colour-Doppler aufgrund des sequentiellen Abtastens besteht. Ausserdem erlaubt die senkrechte Lage der Messschicht, den Volumenfluss unabhängig vom Einstrahlwinkel zu ermitteln [3].

Um das Verfahren auszustesten, wurde ein Laborgrät mit folgenden Daten gebaut: Transducer $16 \times 32 \mathrm{~mm}$ unterteilt in $7 \mathrm{x} 9$ Elemente, $4 \mathrm{MHz}, 4$ Sender zur Ezeugung des breiten Sendepulses, max. 64 Empfangskanäle (63 werden gebraucht) Digitalisierung mit 12 Bit, festverdrahteter Beamformer und FFT-Prozessor für die synthetische Fokussierung bzw. die Frequenzanalyse. Fig. 5 zeigt eine Sequenz solcher Strömungsfelder, gemessen am Flussmodell.
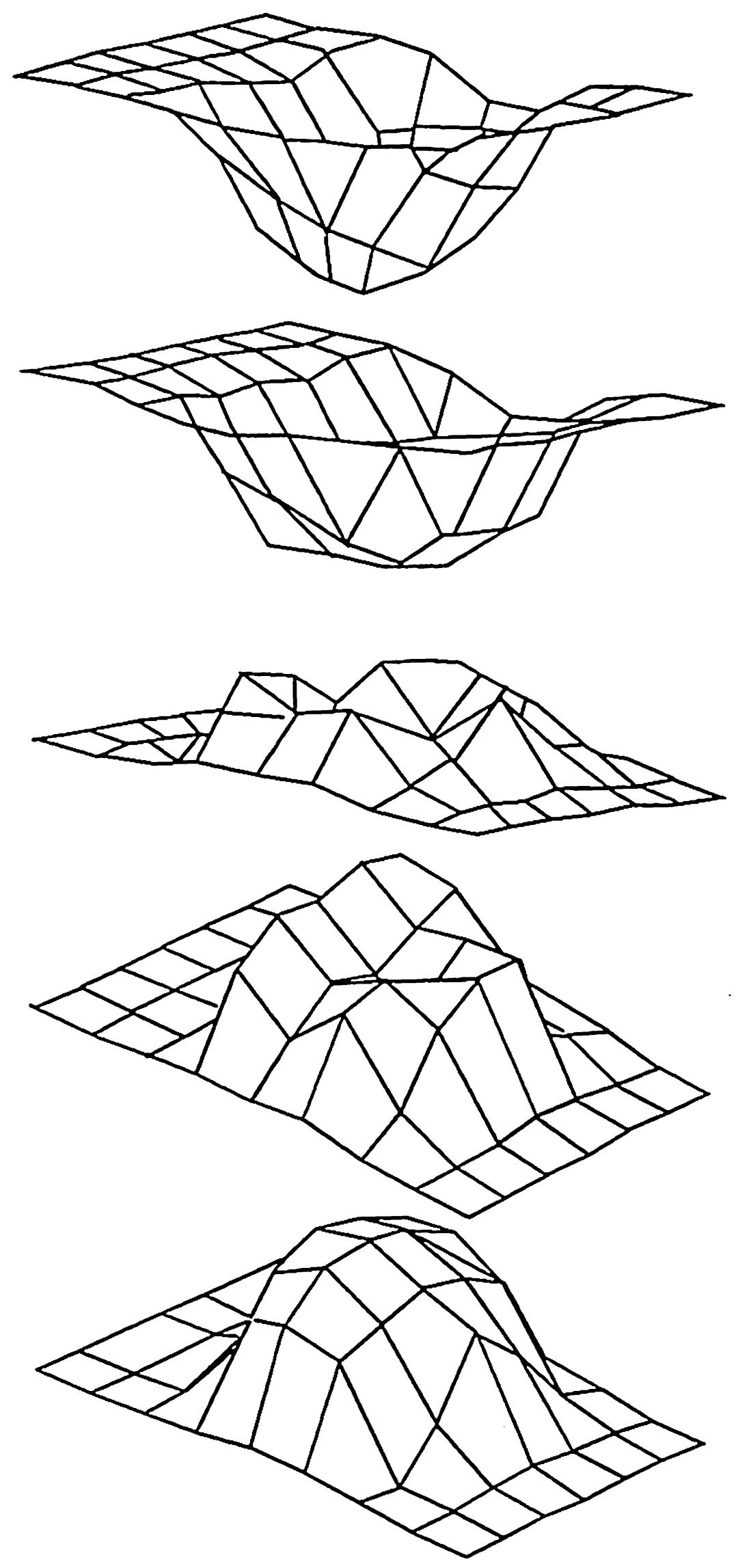

Fig. 5: Mittels C-mode Doppler gemessene Sequenz von 2d-Strömungsfeldern in einem $10 \mathrm{~mm}$ Rohr. Der Fluss wurd von -40 auf $+40 \mathrm{ml} / \mathrm{s}$ umgestellt. Zeitliche Sequenz der Bilder: $30 \mathrm{~ms}$. Transducer 7×9, 16x32mm, $4 \mathrm{MHz}$.
6.Grober 2d-Arrav für einen Sektorscanner mit mechanischer Strahlauslenkung

Auch in der Echographie könnten 'grobe' 2d-Arrays Vorteile bringen. Um Gitterkeulen zu vermeiden bzw. deren Effekte zu minimalisieren, muss jedoch auf eine elektronische Strahlauslenkung verzichtet werden. Wie beim annulären Array ist deshalb die Strahlauslenkung mechanisch zu bewerkstelligen, was natürlich mit den bekannten Nachteilen verbunden ist. Dafür kann die Anzahl benötigter Arrayelemente dramatisch reduziert werden. Ein brauchbarer Array könnte z.B. aus 16×16 Elementen bestehen und ermöglichte es, das Schallfeld zweidimensional dynamisch zu fokussieren sowie beim Senden mehrere Foci zu verwenden. Zusätzlich zu diesen Möglichkeiten, die ja auch beim annulären Array gegeben sind, ist jedoch eine Korrektur der Laufzeitfehler denkbar. Da sich letztere unsymmetrisch auswirken, ist ein 2d-Array unbedingt notwendig.

\section{Schlussfolgerungen}

Der Einsatz zweidimensionaler Arrays wird die Ultraschallbildqualität und Dopplermessungen in den nächsten Jahren noch markant verbessern. Dabei werden jedoch bis auf weiteres Elementgrössen zum Einsatz kommen, die - mindestens in einer Dimension - ein vielfaches der Wellenlänge betragen, so dass sich die Fertigungsprobleme und Kosten im vernünftigen Rahmen halten.

\section{Literatur}

[1] M. Fink, "Time Reversal of Ultrasonic Fields", IEEE Transactions on Ultrasonics, Ferroelectrics and Frequency Control, Vol. 39, No. 5, Sept. 1992.

[2] Dong-Lai Liu, R. C. Waag, "Time-shift compensation of ultrasonic pulse focus degradation using least-mean-square error estimates of arrival time", J. Acoust. Soc. of America, 95 (1), January 1994.

[3] U. Moser, P.M. Schumacher, M. Anliker, "Benefits and Limitations of the C-mode Doppler Procedure", Acoustical Imaging, Vol 21, pp. 509, Plenum Press, 1995. 